Article

\title{
Srebp-1c/Fgf21/Pgc-1 $\alpha$ Axis Regulated by Leptin Signaling in Adipocytes-Possible Mechanism of Caloric Restriction-Associated Metabolic Remodeling of White Adipose Tissue
}

\author{
Masaki Kobayashi ${ }^{1}{ }^{\oplus}$, Seira Uta ${ }^{1}$, Minami Otsubo ${ }^{1}$, Yusuke Deguchi ${ }^{1}$, Ryoma Tagawa ${ }^{1}$, \\ Yuhei Mizunoe ${ }^{2}(\mathbb{D})$, Yoshimi Nakagawa ${ }^{3}\left(\mathbb{D}\right.$, Hitoshi Shimano ${ }^{2,4,5}$ and Yoshikazu Higami ${ }^{1,6, *}$ \\ 1 Laboratory of Molecular Pathology and Metabolic Disease, Faculty of Pharmaceutical Sciences, \\ Tokyo University of Science, Chiba 278-8510, Japan; kobayashim@rs.tus.ac.jp (M.K.); \\ 3B18508@ed.tus.ac.jp (S.U.); 3a15020@ed.tus.ac.jp (M.O.); 3B16060@ed.tus.ac.jp (Y.D.); \\ tagawar@rs.tus.ac.jp (R.T). \\ 2 Department of Internal Medicine (Endocrinology and Metabolism), Faculty of Medicine, University of \\ Tsukuba, Ibaraki 305-8575, Japan; ymizunoe@md.tsukuba.ac.jp (Y.M.); hshimano@md.tsukuba.ac.jp (H.S.) \\ 3 Division of Complex Biosystem Research, Department of Research and Development, Institute of Natural \\ Medicine, University of Toyama, Toyama 930-0194, Japan; ynaka@inm.u-toyama.ac.jp \\ 4 Life Science Center for Survival Dynamics, Tsukuba Advanced Research Alliance (TARA), \\ University of Tsukuba, Ibaraki 305-8575, Japan \\ 5 AMED-CREST, Japan Agency for Medical Research and Development (AMED), Tokyo 100-1004, Japan \\ 6 Research Institute for Biomedical Sciences, Tokyo University of Science, Chiba 278-8510, Japan \\ * Correspondence: higami@rs.tus.ac.jp; Tel./Fax: +81-4-7121-3676
}

Received: 6 June 2020; Accepted: 8 July 2020; Published: 10 July 2020

\begin{abstract}
Caloric restriction (CR) improves whole body metabolism, suppresses age-related pathophysiology, and extends lifespan in rodents. Metabolic remodeling, including fatty acid (FA) biosynthesis and mitochondrial biogenesis, in white adipose tissue (WAT) plays an important role in the beneficial effects of $\mathrm{CR}$. We have proposed that CR-induced mitochondrial biogenesis in WAT is mediated by peroxisome proliferator-activated receptor $\gamma$ coactivator- $1 \alpha$ (PGC- $1 \alpha)$, which is transcriptionally regulated by sterol regulatory element-binding protein 1c (SREBP-1c), a master regulator of FA biosynthesis. We have also proposed that the CR-associated upregulation of SREBP-1 and PGC- $1 \alpha$ might result from the attenuation of leptin signaling and the upregulation of fibroblast growth factor 21 (FGF21) in WAT. However, the detailed molecular mechanisms remain unclear. Here, we interrogate the regulatory mechanisms involving leptin signaling, SREBP-1c, FGF21, and PGC-1 $\alpha$ using Srebp-1c knockout (KO) mice, mouse embryonic fibroblasts, and 3T3-L1 adipocytes, by altering the expression of SREBP-1c or FGF21. We show that a reduction in leptin signaling induces the expression of proteins involved in FA biosynthesis and mitochondrial biogenesis via SREBP-1c in adipocytes. The upregulation of SREBP-1c activates PGC- $1 \alpha$ transcription via FGF21, but it is unlikely that the FGF21-associated upregulation of PGC- $1 \alpha$ expression is a predominant contributor to mitochondrial biogenesis in adipocytes.
\end{abstract}

Keywords: caloric restriction; fatty acid biosynthesis; mitochondrial biogenesis; adipocyte

\section{Introduction}

It is well known that white adipose tissue (WAT) is involved in the pathogenesis of age-related diseases including type 2 diabetes, atherosclerosis, and other cardiovascular and cerebrovascular 
diseases [1]. It has recently been shown that WAT quality, including adipocyte size, mitochondrial biogenesis, and adipokine expression profile, is a key player in lifespan regulation [2-6].

Caloric restriction $(\mathrm{CR})$ is the most robust, reproducible, and simple experimental manipulation that is capable of improving whole body metabolism, delaying the onset of various age-related pathophysiological changes and extending both median and maximum lifespan in a wide range of organisms [7,8]. Dwarf rodents that demonstrate the suppression of growth hormone/insulin-like growth factor 1 (GH/IGF-1) signaling live longer than their wild-type (Wd) littermates [9]. Since CR suppresses GH/IGF-1 signaling, its beneficial effects are considered to be dependent on the suppression of GH/IGF-1 signaling [10]. However, CR further extends the lifespan of long-lived dwarf rodents that have GH/IGF-1 suppression [11,12]. Therefore, the beneficial effects of CR are also likely to be mediated through a GH/IGF-1-independent mechanism.

To identify the GH/IGF-1-independent mechanism involved in the effects of CR, we compared the gene expression profile of the WAT of long-living dwarf rats bearing an antisense $\mathrm{GH}$ transgene with that of $\mathrm{Wd}$ rats subjected to $\mathrm{CR}$, and we found that $\mathrm{CR}$ upregulated the expression of genes involved in fatty acid (FA) biosynthesis in a GH/IGF-1-independent manner [13]. Sterol regulatory element binding protein-1 (SREBP-1), including its two isoforms, SREBP-1a and -1c, is a master transcriptional regulator of FA biosynthesis [14]. In WAT, SREBP-1c is predominantly expressed, rather than SREBP-1a [15]. Therefore, we applied CR to both Srebp-1c knockout (KO) and WT mice on a B6; 129 S6 background and found that $\mathrm{CR}$ extended lifespan in Wd mice but not in $\mathrm{KO}$ mice. Moreover, $\mathrm{CR}$ upregulated the expression of proteins involved in FA biosynthesis and mitochondrial biogenesis in the WAT of Wd mice but not in KO mice. These findings were observed only in WAT but not in the other tissues, including liver, kidney, quadriceps femoris muscle, and heart [16]. Peroxisome proliferator-activated receptor $\gamma$ coactivator- $1 \alpha$ (PGC-1 $\alpha$ ) is a master transcriptional cofactor for mitochondrial biogenesis [17] and a key regulator of the CR-induced activation of mitochondrial biogenesis [18]. We also found that CR upregulates Pgc-1a mRNA in WAT of Wd mice but not in Srebp-1c KO mice. Moreover, a chromatin immunoprecipitation assay showed that SREBP-1 protein binds to the promoter region of the Pgc-1a gene, as well as the Fasn gene, in mouse embryonic fibroblasts (MEFs) derived from WT mice but not in those from $\mathrm{KO}$ mice. Therefore, we suggested that $\mathrm{CR}$ upregulates FA biosynthesis and mitochondrial biogenesis via SREBP-1c in WAT [16].

Fibroblast growth factor 21 (FGF21), which was initially identified as a hepatokine, is mostly secreted by the liver [19]. Circulating FGF21 binds to the FGF receptor (FGFR) and $\beta$-klotho (KLB) receptor complex in target tissues such as WAT. The binding of FGF21 to its receptors activates downstream signaling, including extracellular signal-regulated kinase (ERK) signaling, which upregulates the expression of genes involved in glucose and lipid metabolism [20-22]. FGF21 expression is negatively regulated by SREBP-1c in hepatocytes [23]. In contrast, FGF21 expression is upregulated by SREBP-1c in WAT and 3T3-L1 adipocytes [24]. FGF21 induces PGC-1 $\alpha$ expression in the liver as an adaptation to starvation [25]. In WAT, FGF21 positively regulates PGC-1 $\alpha$ and PPAR $\gamma$ expression and/or activity via feed-forward autocrine/paracrine loops [26,27]. Moreover, Fgf21 Tg mice live longer than Wd mice and have a similar metabolic phenotype to CR mice [28]. We have also shown that the CR-associated upregulation of PGC-1 $\alpha$ expression is partially mediated through FGF21 in WAT [29]. CR also upregulates PPAR $\gamma$ expression in WAT [29]. In addition, the expression of PGC- $1 \alpha$ is increased as a result of rosiglitazone-induced PPAR $\gamma$ activity in WAT [30].

Leptin, which was the first substance to be identified as an adipokine, is mostly secreted by adipocytes [31]. Circulating leptin binds to the leptin receptor, which is predominantly expressed in the arcuate nucleus of the hypothalamus and reduces appetite and increases energy expenditure via the sympathetic nervous system [32]. However, the leptin receptor is also expressed in other cell types, including adipocytes [33]. It has been reported that leptin treatment downregulates the expression of SREBP-1 and its downstream targets in mouse WAT [34]. In addition, CR reduces leptin secretion by adipocytes, thereby reducing the circulating leptin concentration [35]. These findings raised the 
possibility that $C R$ might suppress leptin signaling via an autocrine/paracrine loop, leading to the SREBP-1-induced upregulation of proteins involved in FA biosynthesis in WAT.

As stated above, the molecular mechanisms of CR-associated metabolic remodeling, including FA biosynthesis and mitochondrial biogenesis, are unclear. In particular, the reciprocal regulatory mechanism that involves SREBP-1, FGF21, and PGC- $1 \alpha$ is complex. In the present study, we aimed to clarify this molecular mechanism, focusing on the expression of the master regulators of FA biosynthesis and mitochondrial biogenesis, SREBP- 1 and PGC- $1 \alpha$, respectively, in adipocytes. To this end, we analyzed the regulation of leptin signaling, SREBP-1c, FGF21, and PGC- $1 \alpha$ in the CR-associated metabolic remodeling of WAT and adipocytes.

\section{Materials and Methods}

\subsection{Animals and the Collection of Mice Embryonic Fibroblasts (MEFs)}

All animal experiments were approved by the Animal Experimentation Committees of Tokyo University of Science (Y17051, Y18060, Y19056) or the University of Tsukuba (19-274). We back-crossed Srebp-1c KO mice on a B6;129S6 background (B6; 129S6-Srebf1 tm1Mbr/J; Jackson Laboratory, Bar Harbor, ME, USA) and C57Bl/6J mice (CLEA Japan, Tokyo, Japan) to obtained Srebp-1c KO mice on a C57Bl/6 background. All the animals were maintained under specific pathogen-free conditions. At 3 months of age, Wd and Srebp-1c KO mice were allocated to two groups: an ad libitum-fed (AL) and a CR (70\% of the energy intake of AL) group. At 10 months of age, four groups of mice (WdAL, WdCR, KOAL, and KOCR) were provided with food $0.5-1 \mathrm{~h}$ prior to turning off the lights in the evening, then they were euthanized under isoflurane anesthesia (Mylan, Canonsburg, PA, USA) 2-3 h later, after which WAT was harvested. The time-course measurement of food intake of the Wd and KO mice that were fed AL, and the body weights of the four groups, are shown in Figure S1.

MEFs were obtained from Srebp-1c KO and Wd mice, and F\&f21 KO and Wd mice on a C57Bl/6 background [36], as previously reported [16]. Briefly, 13-15-day old embryos (E13-15) were collected from pregnant mice of each KO line, minced and trypsinized. MEFs were separated by passing the tryptic digests through a cell strainer.

\subsection{Cell Culture and Reagent Treatment}

3T3-L1 preadipocytes were purchased from the Japanese Collection of Research Bioresources (JCRB) cell bank (Osaka, Japan) and maintained in Dulbecco's modified Eagle's medium (DMEM) containing a low glucose concentration (Wako, Osaka, Japan), 10\% fetal bovine serum (FBS) (Thermo Fisher Scientific, Waltham, MA USA), and 1\% penicillin/streptomycin (P/S) (Sigma-Aldrich, MO, USA). MEFs were maintained in DMEM containing a high glucose concentration (Wako), 10\% FBS, $1 \% \mathrm{P} / \mathrm{S}$, and $0.1 \mu \mathrm{M}$ 2-mercaptoethanol (Sigma). The differentiation of 3T3-L1 preadipocytes or MEFs to mature adipocytes was achieved by using our previous published protocol [37]. In the present study, 3T3-L1 cells or MEFs were used as mature adipocytes 8-12 or 16 days after the induction of differentiation, respectively. PD1730741 (Funakoshi, Tokyo, Japan) was dissolved in DMSO to make a $5 \mathrm{mM}$ solution, and then it was diluted in PBS. Differentiated 3T3-L1 cells (day 7) were treated with 50 nM PD1730741 for $24 \mathrm{~h}$ and then collected.

\subsection{Retrovirus Plasmid Construction}

The construction of the retrovirus plasmids for Srebp-1c and Fgf21 overexpression have been described in our previous reports [16,29]. Srebp-1a cDNA was obtained by PCR using KOD FX Neo (Toyobo, Osaka, Japan) and the following primers: 5'-TTT GGA TCC GCC ACC ATG GAC GAG CTG GCC TT-3' and 5'-TTT GAA TTC TTA CAG GGC CAG GCG GGA-3'. Amplified Srebp-1a fragments were digested with BamHI and EcoRI and subcloned into BamHI- and EcoRI-digested pBluescript II SK (+). Then, this plasmid was digested with BamHI and EcoRI, and the gene sequence was inserted into pMXs-AMNN-Puro (pMXs-AMNN-Srebp-1a-Puro) after it was also digested using the same 
enzymes. The target sequences of the shRNAs against Lepr were designed using the Public TRC Portal website (http://www.broadinstitute.org/rnai/public/seq/search), and the sequences were as follows: 5'-GCT AGG TGT AAA CTG GGA CAT CTC GAG ATG TCC CAG TTT ACA CCT AGC TTT TT-3' and 5'-CGA AAA AGC TAG GTG TAA ACT GGG ACA TCT CGA GAT GTC CCA GTT TAC ACC TAG C-3'. The underlined letters are the sense and antisense target sequences. These oligonucleotides were inserted into BstBI- and PmeI-digested pMXs-puro-mU6 (pMXs-puro-shLeptinR).

\subsection{Retrovirus Vector Preparation}

Retrovirus vectors were generated as reported previously [16]. Briefly, each pMXs-AMNN-Puro plasmid or pMXs-puro-shLeptinR plasmid was transfected into Plat-E cells (kindly provided by T. Kitamura, University of Tokyo, Japan) using the calcium phosphate method. To obtain each overexpressing or shLeptinR-expressing 3T3-L1 cell line, the supernatant from each virus-containing culture was collected after 3 days. The 3T3-L1 cells were infected by incubation in the collected virus-containing supernatant for 2 days, followed by treatment with $2 \mu \mathrm{g} / \mathrm{mL}$ puromycin for a further 5 days. The 3T3-L1 cells overexpressing empty vectors or expressing shRNA targeting GFP (shGFP) were used as control cells.

\subsection{RT-PCR and Semi-Quantitative RT-PCR}

RNA was extracted from WAT and other cell types using ISOGENII (Nippon Gene, Tokyo, Japan). The purified RNA was reverse transcribed using ReverTra Ace ${ }^{\circledR}$ qPCR RT Master Mix (Toyobo) and the cDNAs were then amplified using a CFX Connect ${ }^{\mathrm{TM}}$ Real-time System, Thunderbird SYBR qPCR mix, and the primers for each gene. These procedures were performed according to the manufacturer's protocol. Since the intrinsic expression of Srebp-1c mRNA was very low in 3T3-L1 adipocytes, RT-PCR was not possible. Therefore, we performed conventional PCR and agarose gel electrophoresis of the PCR products, followed by ethidium bromide staining. Fluorescence of the ethidium bromide was visualized using an LAS3000 (Fujifilm, Tokyo, Japan) and data were analyzed using Multigauge software (Fujifilm). Target gene expression data were normalized to Rps18 expression $(n=4)$. The primer pair sequences are shown in Table 1.

Table 1. List of primers for RT-PCR.

\begin{tabular}{|c|c|c|}
\hline & Forward & Reverse \\
\hline Adipoq & 5'-TGC CGA AGA TGA CGT TAC AAC-3' & 5'-CTT CAG CTC CTG TCA TTC CAA C-3' \\
\hline Fasn & $5^{\prime}$-AGC AGG CAC ACA CAA TGG AC-3' & $5^{\prime}$-GAA GAA AGA GAG CCG GTT G-3' \\
\hline Fgf21 & 5'-GAA GCC CAC CTG GAG ATC AG-3' & 5'-CAA AGT GAG GCG ATC CAT AGA G-3' \\
\hline LeptinR & $5^{\prime}$ - CAG TCT TCGG GGA TGT GAA TG-3' & 5'- CAT TGT TTG GCT GTC CCA AG-3' \\
\hline PeriA & 5'-TGG GAA GCA TCG AGA AGG TG-3' & 5'-ATG GTG TGT CGA GAA AGA GTG TTG-3' \\
\hline $\operatorname{Pgc}-1 \alpha$ & 5'-AGA CGG ATT GCC CTC ATT TG-3' & 5'-CAG GGT TTG TTC TGA TCC TGT G-3' \\
\hline Rps18 & $5^{\prime}$-TGC GAG TAC TCA ACA CCA ACA T- $3^{\prime}$ & $5^{\prime}$-CTT TCC TCA ACA CCA CAT GAG C-3' \\
\hline Srebp-1a & 5'-GGC CGA GAT GTG CGA ACT-3' & $5^{\prime}$-TTG TTG ATG AGC TGG AGC ATG T-3' \\
\hline Srebp-1c & $5^{\prime}$-GGA GCC ATG GAT TGC ACA TT-3' & 5'-GGC CCG GGA AGT CAC TGT-3' \\
\hline Tbp & 5'-CCC TCA CAC TCA GAT CAT CTT CTC-3' & 5'-GCC TTG TCC CTT GAA GAG AAC C-3' \\
\hline
\end{tabular}

\subsection{Western Blotting}

Cell lysis and immunoblotting were performed as previously described [37]. Briefly, the collected cells were lysed in lysis buffer (50 mM Tris- $\mathrm{HCl}$ (pH 6.8), 2\% SDS, $3 \mathrm{M}$ urea, 6\% glycerol), boiled for $5 \mathrm{~min}$, and sonicated. Lysates containing $15 \mu \mathrm{g}$ protein were subjected to SDS/PAGE and the proteins were then transferred to nitrocellulose membranes. The membranes were blocked with $2.5 \%$ skim milk and $0.25 \%$ bovine serum albumin in Tris-buffered saline (50 mM Tris- $\mathrm{HCl}$ (pH 7.4) and $150 \mathrm{mM} \mathrm{NaCl}$ ) containing $0.1 \%$ Tween 20 (TTBS) for $60 \mathrm{~min}$ at room temperature, then incubated with appropriate primary antibodies overnight at $4{ }^{\circ} \mathrm{C}$. Primary antibodies against FGF21 (Abcam, Cambridge, UK), PGC-1 $\alpha$ (Sigma, MO, USA AB3242), mitochondrial transcription factor A (TFAM) (Proteintech, Chicago, 
IL, USA, 19998-1-AP), SIRT3 (cell signaling technology (CST), Beverly, MA, USA, \#5490), ACC (CST, \#3662), p-STAT3 (Thermo Fisher Scientific, 44-3804), and LaminB1 (Medical \& Biological Laboratories, Nagoya, Japan, PM064) were used. The membranes were then incubated with an appropriate secondary antibody (a horseradish peroxidase-conjugated $\mathrm{F}\left(\mathrm{ab}^{\prime}\right) 2$ fragment of goat anti-mouse IgG or anti-rabbit IgG; Jackson Immuno Research, West Grove, PA, USA) for $60 \mathrm{~min}$ at room temperature. Thereafter, they were incubated with ImmunoStar LD (Wako), specific protein bands were visualized using an LAS3000 (Fujifilm, Tokyo, Japan), and the data were analyzed using Multigauge software (Fujifilm).

\subsection{Statistical Analysis}

The values presented are means \pm standard deviations (SDs). The data were statistically evaluated using Student's t-test, two-way ANOVA and/or Tukey's test, with R software (Version 3.4.1, $\mathrm{R}$ Foundation for Statistical Computing, Vienna, Austria). $p<0.05$ was considered to represent statistical significance.

\section{Results}

\subsection{Role of SREBP-1c in the Effects of CR on Gene Expression in WAT}

We have reported previously that CR increases the expression of Srebp-1c, Srebp-1a, and Pgc-1a mRNAs in the WAT of Wd B6;129S6 mice but not in KO mice [16]. In the WAT of mice on a C57Bl/6 background, CR also increased the expression of Srebp-1c, Pgc-1a, and Fgf21 mRNAs in Wd mice but not in KO mice (Figure 1A or Figure 1C,D). Similar findings about the CR-associated upregulation of Fgf21 mRNA were observed in B6;129S6 mice (Figure S2). However, in contrast to mice on a B6;129S6 background, CR did not upregulate the expression of Srebp-1a mRNA in either Wd or KO mice on a $\mathrm{C} 57 \mathrm{Bl} / 6$ background (Figure 1B). Overall, it is likely that the CR-associated upregulation of these factors is less exaggerated in $\mathrm{C} 57 \mathrm{bl} / 6$ mice compared with B6;129S6 mice.
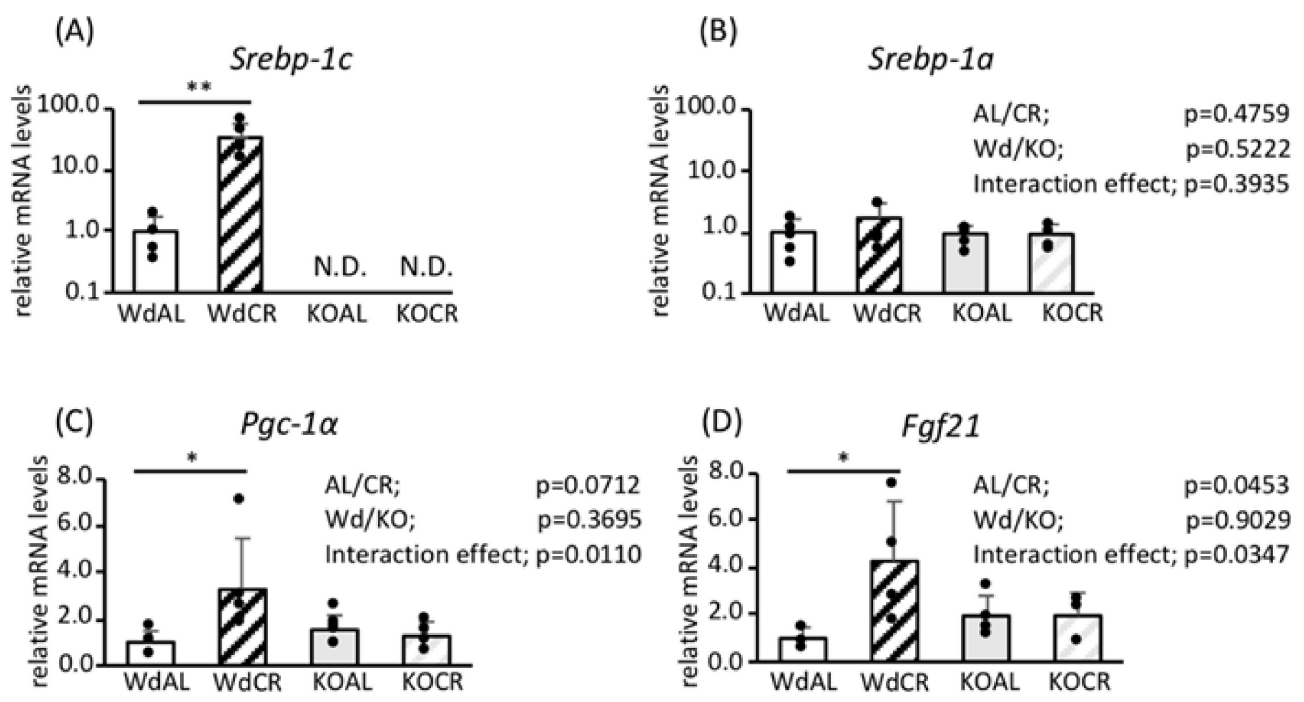

Figure 1. The effects of Srebp-1c KO on the expression of key regulators of CR-associated metabolic remodeling in the WAT of mice on a C57Bl/6 background. The mRNA expression levels of Srebp-1c (A), Srebp-1a (B), Fgf21 (C), and Pgc-1a (D) in WAT were measured using RT-PCR and were normalized to Tbp expression $(n=4)$. Values are means \pm SDs. ${ }^{*} p<0.05,{ }^{* *} p<0.01 \mathrm{vs}$. AL, according to Student's $t$-test, or two-way ANOVA and Tukey's test. 


\subsection{Effects of SREBP-1c on the Expression of Genes and Proteins Involved in FA Biosynthesis and} Mitochondrial Biogenesis

To confirm that SREBP-1c is the significant regulator of the expression of genes and proteins involved in FA biosynthesis and mitochondrial biogenesis in vitro, we generated 3T3-L1 preadipocytes that overexpressed SREBP-1c (SREBP-1c OE) using retroviral vectors. The SREBP-1c OE 3T3-L1 preadipocytes were differentiated to adipocytes (Figure 2A), and the expression levels of mRNAs and proteins of interest were analyzed.
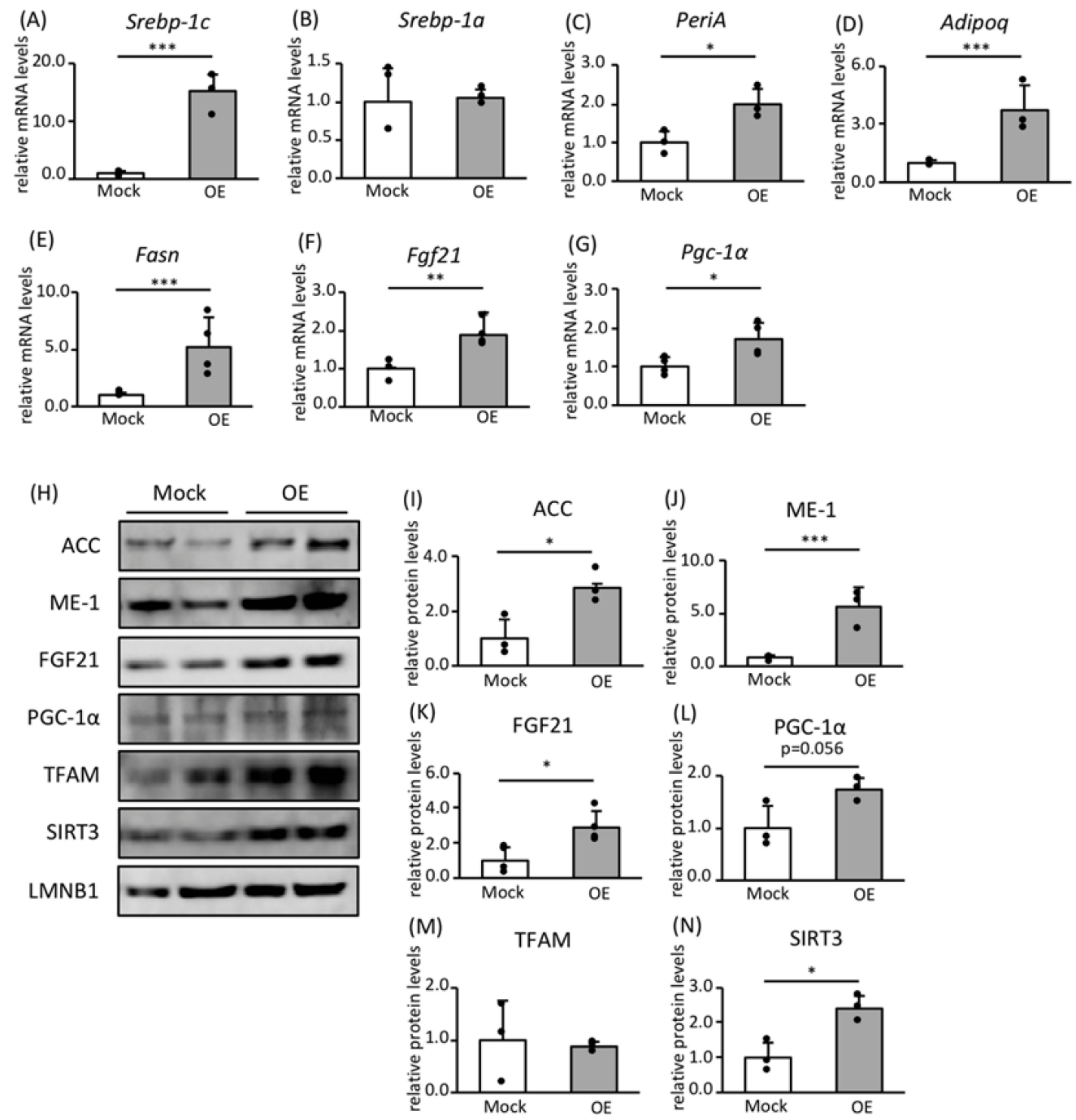

Figure 2. The effects of SREBP-1c overexpression on the expression of genes and proteins involved in fatty acid (FA) biosynthesis and mitochondrial biogenesis in mature 3T3-L1 adipocytes. Control and SREBP-1c OE preadipocytes were differentiated into mature adipocytes in four separate dishes from each phenotype. RNA was extracted and lysates were prepared from each dish. RNA was extracted and lysates were prepared from adipocytes. The mRNA expression levels of Srebp-1c (A), Srebp-1a (B), PeriA (C), Adipoq (D), Fasn (E), Fgf21 (F), and Pgc-1a (G) were determined using RT-PCR and normalized to Rps18 expression $(n=4)$. (H) Representative immunoblot images, showing the expression levels of proteins involved in FA biosynthesis and mitochondrial biogenesis. Quantitative analysis was performed using a chemiluminescence method. The protein expression of ACC (I), ME-1 (J), FGF21 (K), PGC-1 $\alpha(\mathbf{L})$, TFAM $(\mathbf{M})$, and SIRT3 $(\mathbf{N})$ are shown as the relative intensities of the indicated protein divided by that of LMNB1 as an internal control $(n=4)$. Values are means \pm SDs. ${ }^{*} p<0.05,{ }^{* *} p<0.01$, *** $p<0.001$ vs. controls, according to Student's $t$-test. 
In SREBP-1c OE adipocytes, the expression of Srebp-1a mRNA was similar to that of control cells (Figure 2B), whereas that of perilipin A (PeriA) and adiponectin (Adipoq), which are markers of adipocyte differentiation, was upregulated (Figure 2C,D). Fatty acid synthase (FASN) is a rate-limiting enzyme in FA biosynthesis. The expression of Fasn mRNA was high in SREBP-1c OE adipocytes (Figure 2E). Moreover, the expression of both Fgf21 and Pgc-1a mRNAs was high in OE adipocytes (Figure 2F,G). In addition, the protein expression of acetyl-CoA carboxylase (ACC) and malic enzyme 1 (ME1), which are FA biosynthetic enzymes, and FGF21 and PGC-1 $\alpha$ were high in OE adipocytes (Figure 2H-L). With regard to mitochondrial proteins, expression of SIRT3 was also high in SREBP-1c OE adipocytes, but that of TFAM was not (Figure $2 \mathrm{H}$ or Figure 2M,N).

To further characterize the regulation of FA biosynthetic genes by FGF21 and PGC- $1 \alpha$, we generated $\mathrm{Wd}$ and $\mathrm{KO}$ adipocytes by differentiating MEFs derived from $\mathrm{Wd}$ and Srebp-1c KO mice. The expression of Srebp-1c was not detectable in KO adipocytes and that of Srebp-1a was similar to that of control adipocytes (Figure 3A,B). The expression levels of PeriA and Adipoq mRNAs in KO adipocytes did not differ from those in Wd adipocytes, suggesting that SREBP-1c deficiency did not alter differentiation (Figure 3C,D). However, the expression of Fasn was lower in KO adipocytes than Wd adipocytes (Figure 3E). The expression of Fgf21 mRNA was slightly reduced, while that of Pgc-1a mRNA was significantly lower in $\mathrm{KO}$ adipocytes (Figure 3F,G). Taken together, our findings suggest that the expression of both $P g c-1 a$ and $F g f 21$ is positively regulated by Srebp-1c, in addition to that of Fasn. Moreover, SREBP-1c is the significant regulator of the expression of genes and proteins involved in FA biosynthesis and mitochondrial biogenesis in adipocytes.
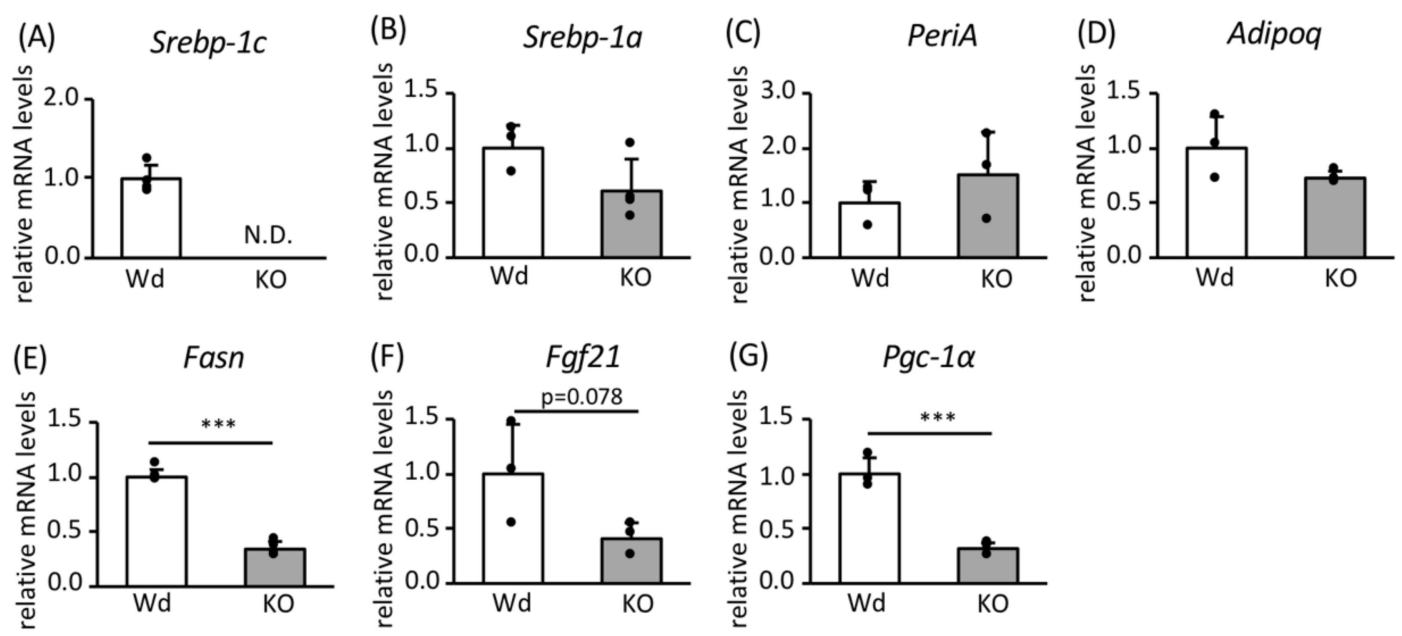

Figure 3. The effects of SREBP-1c deficiency on the expression of adipocyte differentiation markers and genes involved in FA biosynthesis and mitochondrial biogenesis in mature adipocytes. MEFs were obtained from four individual embryos of either $\mathrm{Wd}$ or Srebp-1c KO mice, differentiated to mature adipocytes, and then RNA was extracted from each dish. The mRNA expression levels of Srebp-1c (A), Srebp-1a (B), PeriA (C), Adipoq (D), Fasn (E), Fgf21 (F), and Pgc-1a (G) were determined using RT-PCR and normalized to Rps18 expression $(n=4)$. Values are means \pm SDs. ${ }^{* * *} p<0.001 \mathrm{vs.} \mathrm{Wd,} \mathrm{according} \mathrm{to}$ Student's $t$-test.

\subsection{Roles of FGF21 and PGC-1 $\alpha$ in Mitochondrial Biogenesis}

CR upregulated the expression of both $F g f 21$ and $P g c-1 \alpha$ mRNAs and proteins via SREBP-1c. Therefore, we next determined the roles of FGF21 and PGC-1 $\alpha$ in mitochondrial biogenesis.

In FGF21 OE adipocytes (Figure 4A or Figure 4C,D), the expression of Pgc-1 $\alpha$ mRNA and PGC-1 $\alpha$ protein was high (Figure 4B,C or Figure 4E,F). Treatment with PD173074, an FGF receptor (FGFR) inhibitor, reduced the phosphorylation of ERK without reducing Fgf21 mRNA and FGF21 protein 
expression (Figure $4 \mathrm{~A}$ or Figure $4 \mathrm{C}-\mathrm{E}$ ). In addition, this treatment did not reduce the expression of $P g c-1 \alpha$ mRNA or PGC-1 $\alpha$ protein (Figure 4B,C or Figure 4F).

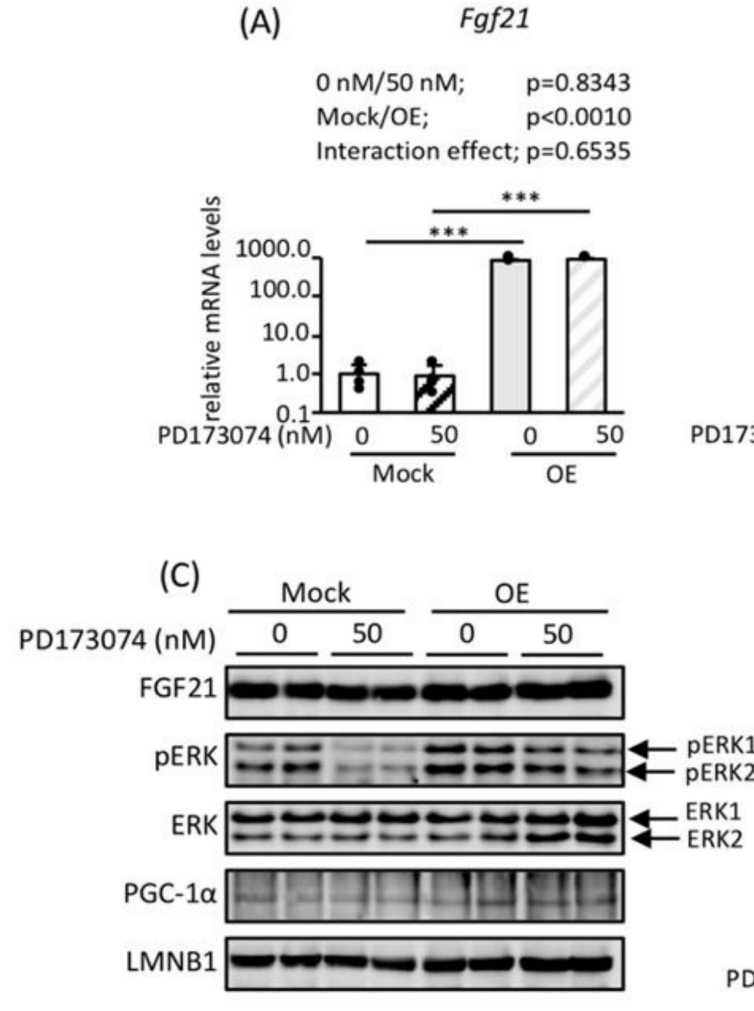

(E)

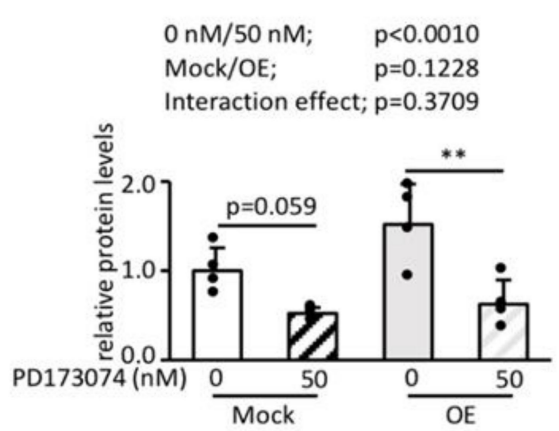

(B) $\quad P g c-1 \alpha$

$\begin{array}{ll}0 \mathrm{nM} / 50 \mathrm{nM} ; & \mathrm{p}=0.2818 \\ \text { Mock/OE; } & \mathrm{p}=0.0015\end{array}$

Interaction effect; $p=0.8876$

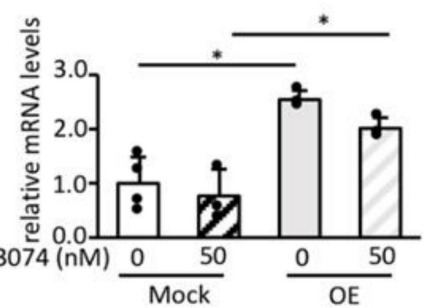

(D)

FGF21

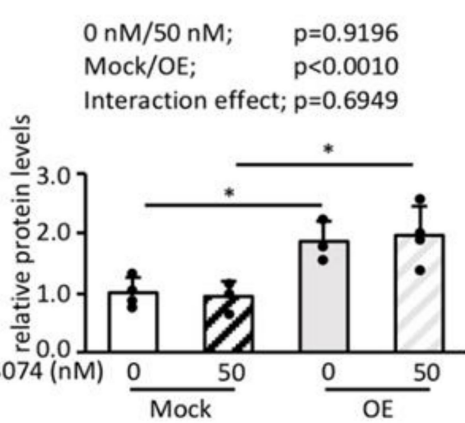

(F)

PGC-1 $\alpha$

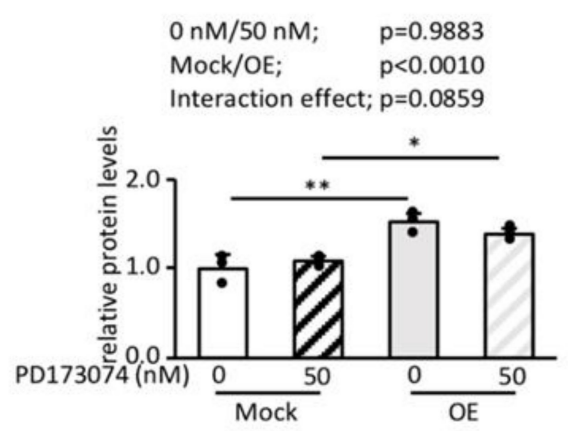

Figure 4. The effects of FGF21 overexpression and the inhibition of FGFR on the expression of genes and proteins involved in FGF21 signaling and PGC-1 $\alpha$ in mature 3T3-L1 adipocytes. Control and FGF21 OE preadipocytes were differentiated into mature adipocytes in four separate dishes for each phenotype, and they were treated with or without $50 \mathrm{nM}$ PD173074, an FGFR inhibitor, for $24 \mathrm{~h}$. $(\mathbf{A}, \mathbf{B})$ RNA was extracted and lysates were prepared from each dish. The mRNA expression levels of Fgf21 (A) and Pgc-1a (B) were determined using RT-PCR and normalized to Rps18 expression $(n=4)$. (C) Representative immunoblot images showing the expression of proteins involved in FGF21 signaling and mitochondrial biogenesis. Quantitative analysis was performed using a chemiluminescence method. The protein expression of FGF21 (D) and PGC-1 $\alpha(\mathbf{F})$ is shown as the relative intensity of the indicated protein divided by that of LMNB1 as an internal control $(n=4)$. Extracellular signal-regulated kinase (ERK) phosphorylation is expressed as the relative intensity of the phosphorylated form of $\operatorname{ERK} /$ total ERK $(n=4)(E)$. Values are means \pm SDs ${ }^{*} p<0.05,{ }^{* *} p<0.01,{ }^{* * *} p<0.001$ vs. controls administered the same treatment. 
In Fgf21 KO adipocytes differentiated from MEFs, the expression levels of PeriA, Adipoq, and Pgc-1 $\alpha$ mRNAs were much lower than in control cells, suggesting that the significant reduction in Pgc-1 $\alpha$ mRNA expression is associated with impaired adipocyte differentiation (Figure 5A-D).
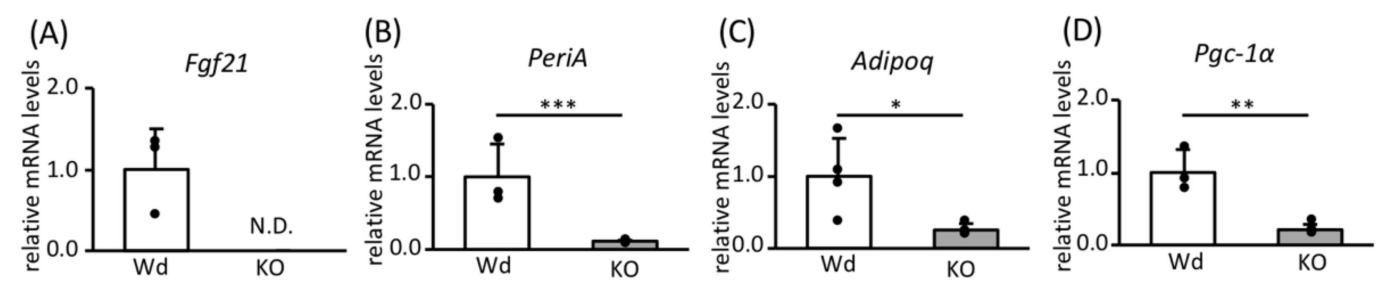

Figure 5. The effects of FGF21 deficiency on the expression of adipocyte differentiation markers and genes involved in mitochondrial biogenesis in mature adipocytes. MEFs were obtained from four individual embryos of either Wd or Fgf $21 \mathrm{KO}$ mice, differentiated to mature adipocytes, and then RNA was extracted from each dish. The mRNA expression levels of Fgf21 (A), PeriA (B), Adipoq (C), and $P g c-1 a(\mathbf{D})$ were analyzed using RT-PCR and normalized to Rps18 expression $(n=4)$. Values are means \pm SDs. ${ }^{*} p<0.05,{ }^{* *} p<0.01$ vs. Wd, according to Student's $t$-test, ${ }^{* * *} p<0.001$.

Taken together, these findings indicate that FGF21 positively regulates PGC-1 $\alpha$ expression, but ERK signaling does not have a significant effect.

\subsection{Effect of Leptin Signaling on the Expression of Genes and Proteins Involved in FA Biosynthesis and Mitochondrial Biogenesis}

To determine the effect of leptin signaling on FA biosynthesis and mitochondrial biogenesis in adipocytes, we knocked down leptin receptor expression in 3T3-L1 preadipocytes using a retroviral vector and analyzed the cells after differentiating them to adipocytes (Figure 6A). The activation of the leptin receptor phosphorylates STAT3, the major downstream target molecule of leptin signaling [38]. Leptin receptor knockdown (KD) reduced the phosphorylation of STAT3, confirming that leptin signaling had been inhibited (Figure 6F,G). The expression of Srebp-1c, Srebp-1a, Fgf21, and Pgc-1 $\alpha$ mRNAs was significantly higher in leptin receptor KD adipocytes than in control cells (Figure 6B-E). Moreover, the expression of FGF21, PGC- $1 \alpha$, TFAM, ACC, and ME-1 proteins was higher in leptin receptor KD adipocytes (Figure $6 \mathrm{~F}$ or Figure $6 \mathrm{H}-\mathrm{L}$ ). This finding suggests that a reduction in leptin signaling induces the expression of both Srebp-1c and Srebp-1a mRNAs and the expression of proteins involved in FA biosynthesis and mitochondrial biogenesis in adipocytes. 

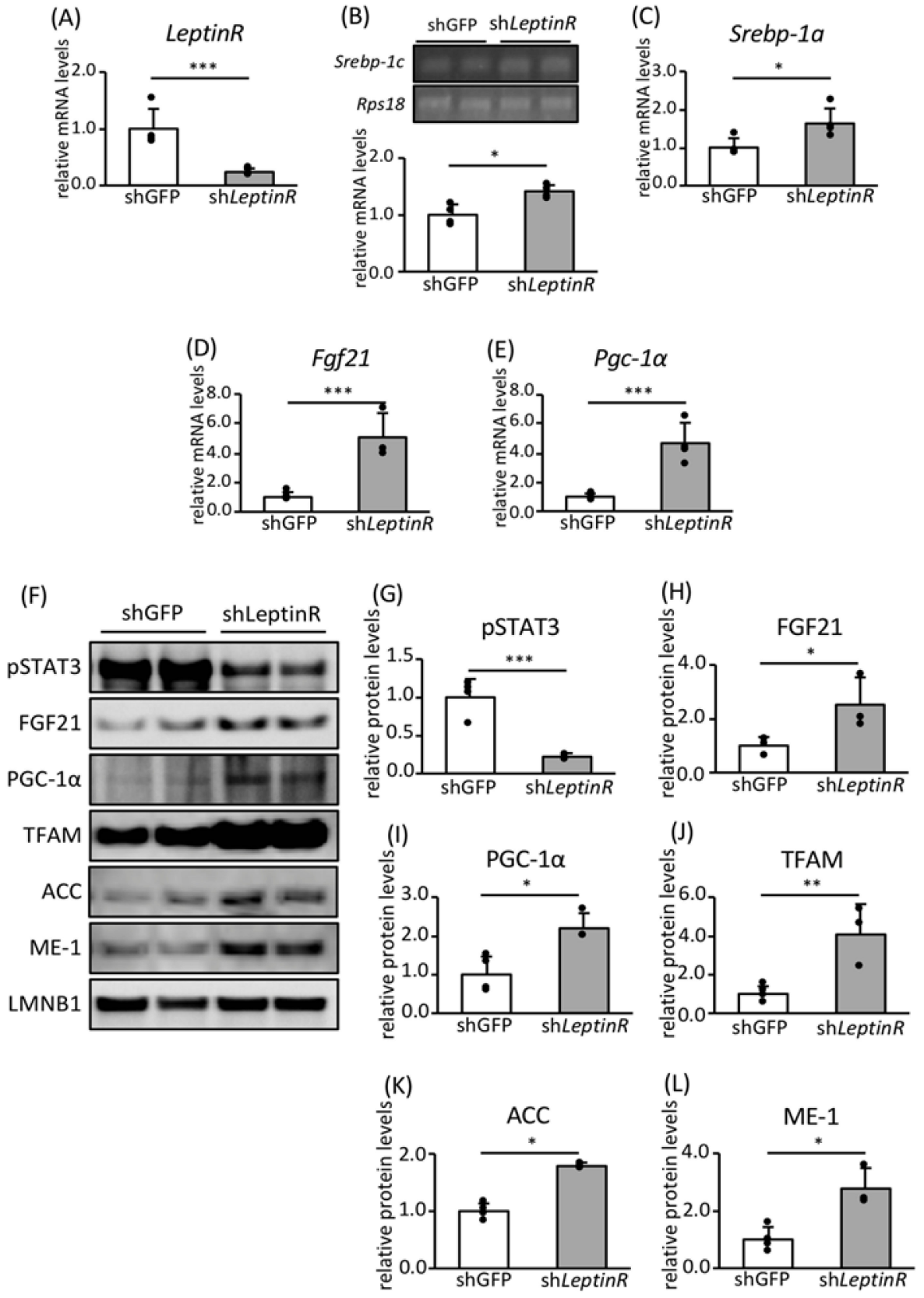

Figure 6. The effects of leptin signaling on the expression of genes and proteins involved in leptin signaling, FA biosynthesis, and mitochondrial biogenesis in mature 3T3-L1 adipocytes. LeptinR knockdown (KD) (shLeptinR) and control (shGFP) preadipocytes were differentiated into mature adipocytes in four separate dishes for each phenotype, and then RNA was extracted, and lysates were prepared from each dish. The mRNA expression levels of LeptinR (A), Srebp-1a (C), Fgf21 (D), and Pgc-1a (E) were determined using RT-PCR and normalized to Rps18 expression $(n=4)$. (B) Representative images of ethidium bromide-stained gels, showing fluorescence corresponding to the products of Srebp-1c cDNA amplification by RT-PCR. Semiquantitative analysis was performed and the data were normalized to Rps18 expression $(\mathrm{n}=4)$. (F) Representative immunoblot images showing the expression of proteins involved in leptin signaling, FA biosynthesis, and mitochondrial biogenesis. Quantitative analysis was performed using a chemiluminescence method. The protein expression of pSTAT (G), FGF21 (H), PGC-1 $\alpha(\mathbf{I})$, TFAM (J), ACC (K), and ME-1 (L) is shown as the relative intensity of the indicated protein divided by that of LMNB1 as an internal control $(n=4)$. Values are means \pm SDs. ${ }^{*} p$ $<0.05,{ }^{* *} p<0.01,{ }^{* * *} p<0.001$ vs. shGFP, according to Student's $t$-test. 


\section{Discussion}

SREBP-1 is a family of transcription factors that are master regulators of FA biosynthesis. It comprises two isoforms, SREBP-1c and SREBP-1a. SREBP-1c is upregulated in the livers of obese mice [39,40], and fatty liver occurs in mice with liver-specific overexpression of SREBP-1c [41]. According to previous findings, SREBP-1c functions predominantly in the liver, rather than in WAT, and is involved in hepatic steatosis [42,43]. However, we have reported previously that CR upregulates the expression of genes and/or proteins involved in FA biosynthesis and mitochondrial biogenesis, including Pgc-1a mRNA expression via SREBP-1c, in WAT rather than in the livers of mice on a B6;129S6 background [16]. In the present study, we have shown similar results in mice on a C57B1/6 background and mature adipocytes differentiated from MEFs. We also demonstrated that CR upregulates FGF21 via SREBP-1c in mice on both B6;129S6 and C57Bl/6 backgrounds. Fgf21 and Pgc-1a mRNA transcripts were downregulated in adipocytes differentiated from MEFs derived from SREBP1c KO mice, but these gene expressions were unchanged in WAT between WdAL and KOAL mice. We are not able to explain the discrepancy between the in vitro and in vivo findings, but these results reveal, at least, that SREBP-1c positively regulates both gene expressions. We also generated SREBP-1a OE 3T3-L1 adipocytes (Figure S3A) and the expression levels of mRNAs and proteins of interest were analyzed. Since the intrinsic expression of Srebp-1c mRNA was very low in 3T3-L1 adipocytes, RT-PCR was not possible. In SREBP-1a OE adipocytes, the expression of Srebp-1c mRNA was similar in control and SREBP-1a OE adipocytes (Figure S3B). However, the expression of PeriA and Adipoq mRNA was high in OE cells (Figure S3C,D), as was that of Fasn and Fgf21 mRNAs, but this was not the case for Pgc-1a mRNA (Figure S3E-G). In addition, the protein expression of ACC, ME-1, FGF21, PGC-1 $\alpha$, and SIRT3 was unaffected or downregulated by OE (Figure S3H-N). In contrast, protein expression of TFAM was high in SREBP-1a OE adipocytes but not in SREBP-1c adipocytes. Moreover, Fgf 21 mRNA expression was upregulated in both SREBP-1c and -1a OE 3T3-L1 adipocytes. In contrast, FGF21 protein was increased in SREBP-1C OE adipocytes but decreased in SREBP-1a OE adipocytes. We are not able to rationally explain these distorted results. However, overall, by comparing SREBP-1c and SREBP-1a OE 3T3-L1 adipocytes, we have confirmed that SREBP-1c, rather than SREBP-1a, is principally responsible for increases in the expression of genes and proteins involved in FA biosynthesis in mature adipocytes.

It has been reported that $\mathrm{CR}$ activates mitochondrial biogenesis in various tissues, including WAT, liver, heart, and skeletal muscle [44,45]. However, we found that CR-induced mitochondrial biogenesis is mediated by SREBP-1c only in WAT [16]. ME-1, which is upregulated in SREBP-1c OE adipocytes, is one of the enzymes involved in the pyruvate/malate cycle. Our previous proteomic analysis showed that $C R$ upregulates the expression of proteins involved in the pyruvate/malate cycle, including ATP-citrate lyase, citrate synthase, mitochondrial pyruvate dehydrogenase E1 component subunit beta, and mitochondrial pyruvate carboxylase, as well as ME-1. Therefore, we hypothesized that CR might activate the pyruvate/malate cycle in WAT in order to switch from the use of glucose to the use of energy-dense FAs so that energy can be used more efficiently under poor food supply conditions [46]. On the basis of this hypothesis, it makes sense that CR would simultaneously upregulate the expression of proteins involved in FA biosynthesis, the pyruvate/malate cycle, and mitochondrial biogenesis via SREBP-1c. Bruss et al. has shown that CR activates de novo FA biosynthesis predominantly in WAT, rather than in the liver [47], and their findings are consistent with this hypothesis.

FGF21 positively regulates PGC- $1 \alpha$ and PPAR $\gamma$ via feed-forward autocrine/paracrine loops in WAT $[26,27]$. It is widely accepted that PGC- $1 \alpha$ is a master regulator of CR-associated mitochondrial biogenesis [18]. We have shown here that CR upregulates the expression of both FGF21 and PGC-1 $\alpha$ via SREBP-1c. CR also upregulates PPAR $\gamma$ expression in WAT [29]. Therefore, we characterized the reciprocal regulatory mechanism involving FGF21 and PGC-1 $\alpha$ expression in mitochondrial biogenesis. In F $g f 21 \mathrm{KO}$ adipocytes, the expression of PGC- $1 \alpha$ was low and adipocyte differentiation was impaired. However, in FGF21 OE adipocytes, the expression of Pgc-1a mRNA and PGF-1 $\alpha$ protein was high [29]. FGF21 promotes the phosphorylation of ERK via the binding of FGF21 to FGFR and the $\beta$-klotho (KLB) receptor complex [21]. Treatment with an FGFR inhibitor reduced the phosphorylation of ERK 
and the expression of Pgc-1a mRNA but not that of PGC-1 $\alpha$ protein. In SREBP-1c OE adipocytes, the expression of FGF21, PGC-1 $\alpha$, and SIRT3 proteins was very high. We previously demonstrated that SREBP-1c binds to the promoter of the Pgc-1a gene in adipocytes derived from Wd MEFs, but this did not occur in SREBP-1c KO MEFs [16]. Furthermore, in brown adipocytes, it has been reported that SREBP-1c activates the Pgc-1a promoter [48]. Therefore, when CR induces PGC-1 $\alpha$ expression in adipocytes, it is likely that direct transcriptional regulation by SREBP-1c is more significant than the induction of FGF21 by SREBP-1c.

Leptin is secreted by WAT and acts as a satiety signal to the hypothalamus, activating NPY and AGRP neurons and suppressing POMC and CART neurons in the hypothalamus, subsequently activating the sympathetic nervous system and thereby lipolysis in WAT via $\beta 3$-adrenergic receptors [32]. We have shown that a reduction in leptin signaling increases the expression of SREBP-1c, SREBP-1a, SREBP-1-regulated genes, FGF 21, and PGC-1 $\alpha$ in mature adipocytes. These findings suggest that lower leptin secretion reduces leptin receptor signaling via an autocrine/paracrine loop, resulting in the greater expression of genes involved in FA biosynthesis and mitochondrial biogenesis in the WAT of CR mice. Previously, we found that the expression of proteins involved in FA biosynthesis is higher in obese fa/fa Zucker rats that have a leptin receptor mutation than in lean $+/+$ rats. Moreover, CR increases the expression of proteins involved in FA biosynthesis in lean +/+ rats but not in obese $\mathrm{fa} / \mathrm{fa}$ Zucker rats [49]. Our present in vitro findings are consistent with these findings in Zucker rats.

Based on findings concerning the CR-associated metabolic remodeling of WAT in Srebp-1c KO mice, we investigated the upstream and downstream regulatory mechanisms of SREBP-1c in vitro. To confirm our results in vitro, we examined the mRNA and protein levels of most factors in both OE cells and $\mathrm{KD}$ or $\mathrm{KO}$ cells, and we were able to obtain relatively consistent data with regard to upregulated and downregulated genes. As a result, it was likely that a reduction in leptin signaling induced the expression of proteins involved in FA biosynthesis and mitochondrial biogenesis via SREBP-1c in adipocytes. PGC- $1 \alpha$ is upregulated via both the direct transcriptional regulation of SREBP-1c and the upregulation of FGF21 indirectly regulated by SREBP-1c, but it is unlikely that the FGF21-associated upregulation of PGC- $1 \alpha$ expression is a predominant factor in mitochondrial biogenesis induced by SREBP-1c. Therefore, we conclude that CR might downregulate an autocrine/paracrine loop involving leptin, with a reduction in leptin signaling activating de novo FA biosynthesis and mitochondrial biogenesis through the upregulation of SREBP-1c in WAT, in addition to the effects that leptin exerts via the central nervous system. SREBP-1c expression is high when the leptin concentration is low, and this is regulated in a GH/IGF-1-independent manner, but it is a key player in the CR-associated metabolic remodeling of WAT, which involves the upregulation of both FA biosynthesis and mitochondrial biogenesis. The CR-associated metabolic remodeling of WAT might be a leptin-mediated adaptive response to food shortage, causing a switch from the use of glucose to lipid as an energy substrate.

Supplementary Materials: The following is available online at http://www.mdpi.com/2072-6643/12/7/2054/s1, Figure S1: Food intake and body weight in Srebp-1c KO and Wd mice fed AL and subjected to CR. Figure S2: The effects of Srebp-1c KO on Fgf21 expression in the WAT of mice on a C57Bl/6-129S6 background. Figure S3: The effects of SREBP-1a overexpression on the expression of genes and proteins involved in FA biosynthesis and mitochondrial biogenesis in mature 3T3-L1 adipocytes.

Author Contributions: Conceptualization, Y.H.; formal analysis, M.K.; methodology, M.K. and Y.H.; investigation, S.U., M.O., Y.D., R.T., and Y.M.; resources, M.K., Y.N., H.S., and Y.H.; writing-original draft preparation, M.K. and Y.H.; writing-review and editing, M.K. and Y.H.; supervision, M.K. and Y.H.; project administration, Y.H.; funding acquisition, Y.H. All authors have read and agreed to the published version of the manuscript.

Funding: This work was supported by Grants-in-Aid for Scientific Research (B) (No. 17H02179) from the Japan Society for the Promotion of Science.

Acknowledgments: We thank Mark Cleasby, from Edanz Group (https://en-author-services.edanzgroup.com/) for editing a draft of this manuscript.

Conflicts of Interest: All authors declare no conflict of interest. 


\section{References}

1. Ouchi, N.; Parker, J.L.; Lugus, J.J.; Walsh, K. Adipokines in inflammation and metabolic disease. Nat. Rev. Immunol. 2011, 11, 85-97. [CrossRef]

2. Blüher, M.; Kahn, B.B.; Kahn, C.R. Extended longevity in mice lacking the insulin receptor in adipose tissue. Science 2003, 299, 572-574. [CrossRef] [PubMed]

3. Otabe, S.; Yuan, X.; Fukutani, T.; Wada, N.; Hashinaga, T.; Nakayama, H.; Hirota, N.; Kojima, M.; Yamada, K. Overexpression of human adiponectin in transgenic mice results in suppression of fat accumulation and prevention of premature death by high-calorie diet. Am. J. Physiol. Endocrinol. Metab. 2007, 293, 2102-2118. [CrossRef] [PubMed]

4. Farmer, S.R. Transcriptional control of adipocyte formation. Cell Metab. 2006, 4, 263-273. [CrossRef] [PubMed]

5. Chiu, C.H.; Lin, W.D.; Huang, S.Y.; Lee, Y.H. Effect of a C/EBP gene replacement on mitochondrial biogenesis in fat cells. Genes Dev. 2004, 18, 19701-19975. [CrossRef] [PubMed]

6. Argmann, C.; Dobrin, R.; Hekkinen, S.; Auburtin, A.; Pouilly, L.; Cock, T.; Koutnikova, H.; Zhu, J.; Schadt, E.E.; Auwerx, J. Ppary2 is a key driver of longevity in the mouse. PLoS Genet. 2009, 5, e1000752. [CrossRef] [PubMed]

7. Masoro, E.J. Overview of caloric restriction and ageing. Mech. Ageing Dev. 2005, 126, 913-922. [CrossRef]

8. Sohal, R.S.; Weindruch, R. Oxidative stress, caloric restriction, and aging. Science 1996, 273, 59-63. [CrossRef]

9. Bartke, A.; Quainoo, N. Impact of growth hormone-related mutations on mammalian aging. Front. Genet. 2018, 9, 586. [CrossRef]

10. Bonkowski, M.S.; Rocha, J.S.; Masternak, M.M.; Al-Regaiey, K.A.; Bartke, A. Targeted disruption of growth hormone receptor interferes with the beneficial actions of calorie restriction. Proc. Natl. Acad. Sci. USA 2006, 103, 7901-7905. [CrossRef]

11. Sun, L.Y.; Spong, A.; Swindell, W.R.; Fang, Y.; Hill, C.; Huber, J.A.; Boehm, J.D.; Westbrook, R.; Salvatori, R.; Bartke, A. Growth hormone-releasing hormone disruption extends lifespan and regulates response to caloric restriction in mice. Elife 2013, 2, e01098. [CrossRef] [PubMed]

12. Shimokawa, I.; Higami, Y.; Tsuchiya, T.; Otani, H.; Komatsu, T.; Chiba, T.; Yamaza, H. Life span extension by reduction of the growth hormone-insulin-like growth factor-1 axis: Relation to caloric restriction. FASEB J. 2003, 17, 1108-1109. [CrossRef] [PubMed]

13. Chujo, Y.; Fujii, N.; Okita, N.; Konishi, T.; Narita, T.; Yamada, A.; Haruyama, Y.; Tashiro, K.; Chiba, T.; Shimokawa, I.; et al. Caloric restriction-associated remodeling of rat white adipose tissue: Effects on the growth hormone/insulin-like growth factor-1 axis, sterol regulatory element binding protein-1, and macrophage infiltration. Age 2013, 35, 1143-1156. [CrossRef] [PubMed]

14. Osborne, T.F.; Espenshade, P.J. Evolutionary conservation and adaptation in the mechanism that regulates SREBP action: What a long, strange tRIP it's been. Genes Dev. 2009, 23, 2578-2591. [CrossRef] [PubMed]

15. Shimomura, I.; Shimano, H.; Horton, J.D.; Goldstein, J.L.; Brown, M.S. Differential expression of exons 1a and 1c in mRNAs for sterol regulatory element binding protein-1 in human and mouse organs and cultured cells. J. Clin. Investig. 1997, 99, 838-845. [CrossRef]

16. Fujii, N.; Narita, T.; Okita, N.; Kobayashi, M.; Furuta, Y.; Chujo, Y.; Sakai, M.; Yamada, A.; Takeda, K.; Konishi, T.; et al. Sterol regulatory element-binding protein-1c orchestrates metabolic remodeling of white adipose tissue by caloric restriction. Aging Cell 2017, 16, 508-517. [CrossRef]

17. Scarpulla, R.C. Metabolic control of mitochondrial biogenesis through the PGC-1 family regulatory network. Biochim. Biophys. Acta (BBA) Mol. Cell Res. 2011, 1813, 1269-1278. [CrossRef]

18. Anderson, R.M.; Barger, J.L.; Edwards, M.G.; Braun, K.H.; O'Connor, C.E.; Prolla, T.A.; Weindruch, R. Dynamic regulation of PGC-1alpha localization and turnover implicates mitochondrial adaptation in calorie restriction and the stress response. Aging Cell 2008, 7, 101-111. [CrossRef]

19. Nishimura, T.; Nakatake, Y.; Konishi, M.; Itoh, N. Identification of a novel FGF, FGF-21, preferentially expressed in the liver. Biochim. Biophys. Acta (BBA) Gene Struct. Expr. 2000, 1492, 203-206. [CrossRef]

20. Ding, X.; Boney-Montoya, J.; Owen, B.M.; Bookout, A.L.; Coate, K.C.; Mangelsdorf, D.J.; Kliewer, S.A. $\beta K$ lotho is required for fibroblast growth factor 21 effects on growth and metabolism. Cell Metab. 2012, 16, 387-393. [CrossRef] 
21. Ogawa, Y.; Kurosu, H.; Yamamoto, M.; Nandi, A.; Rosenblatt, K.P.; Goetz, R.; Eliseenkova, A.V.; Mohammadi, M.; Kuro-o, M. BetaKlotho is required for metabolic activity of fibroblast growth factor 21. Proc. Natl. Acad. Sci. USA 2007, 104, 7432-7437. [CrossRef] [PubMed]

22. Markan, K.R.; Naber, M.C.; Ameka, M.K.; Anderegg, M.D.; Mangelsdorf, D.J.; Kliewer, S.A.; Mohammadi, M.; Potthoff, M.J. Circulating FGF21 is liver derived and enhances glucose uptake during refeeding and overfeeding. Diabetes 2014, 63, 4057-4063. [CrossRef]

23. Zhang, Y.; Lei, T.; Huang, J.F.; Wang, S.B.; Zhou, L.L.; Yang, Z.Q.; Chen, X.D. The link between fibroblast growth factor 21 and sterol regulatory element binding protein 1c during lipogenesis in hepatocytes. Mol. Cell Endocrinol. 2011, 342, 414-417. [CrossRef] [PubMed]

24. Véniant, M.M.; Hale, C.; Helmering, J.; Chen, M.M.; Stanislaus, S.; Busby, J.; Vonderfecht, S.; Xu, J.; Lloyd, D.J. FGF21 promotes metabolic homeostasis via white adipose and leptin in mice. PLoS ONE 2012, 7, e40164. [CrossRef]

25. Potthoff, M.J.; Inagaki, T.; Satapati, S.; Ding, X.; He, T.; Goetz, R.; Mohammadi, M.; Finck, B.N.; Mangelsdorf, D.J.; Kliewer, S.A.; et al. FGF21 induces PGC-1alpha and regulates carbohydrate and fatty acid metabolism during the adaptive starvation response. Proc. Natl. Acad. Sci. USA 2009, 106, 10853-10858. [CrossRef] [PubMed]

26. Fisher, F.M.; Kleiner, S.; Douris, N.; Fox, E.C.; Mepani, R.J.; Verdeguer, F.; Wu, J.; Kharitonenkov, A.; Flier, J.S.; Maratos-Flier, E.; et al. FGF21 regulates PGC-1 $\alpha$ and browning of white adipose tissues in adaptive thermogenesis. Genes Dev. 2012, 26, 2712-2781. [CrossRef] [PubMed]

27. Dutchak, P.A.; Katafuchi, T.; Bookout, A.L.; Choi, J.H.; Yu, R.T.; Mangelsdorf, D.J.; Kliewer, S.A. Fibroblast growth factor-21 regulates PPAR $\gamma$ activity and the antidiabetic actions of thiazolidinediones. Cell 2012, 148, 556-567. [CrossRef]

28. Zhang, Y.; Xie, Y.; Berglund, E.D.; Coate, K.C.; He, T.T.; Katafuchi, T.; Xiao, G.; Potthoff, M.J.; Wei, W.; Wan, Y.; et al. The starvation hormone, fibroblast growth factor-21, extends lifespan in mice. Elife 2012, 1, e00065. [CrossRef]

29. Fujii, N.; Uta, S.; Kobayashi, M.; Sato, T.; Okita, N.; Higami, Y. Impact of aging and caloric restriction on fibroblast growth factor 21 signaling in rat white adipose tissue. Exp. Gerontol. 2019, 118, 55-64. [CrossRef]

30. Wilson-Fritch, L.; Nicoloro, S.; Chouinard, M.; Lazar, M.A.; Chui, P.C.; Leszyk, J.; Straubhaar, J.; Czech, M.P.; Corvera, S. Mitochondrial remodeling in adipose tissue associated with obesity and treatment with rosiglitazone. J. Clin. Investig. 2004, 114, 1281-1289. [CrossRef]

31. Zhang, Y.; Proenca, R.; Maffei, M.; Barone, M.; Leopold, L.; Friedman, J.M. Positional cloning of the mouse obese gene and its human homologue. Nature 1994, 372, 425-432. [CrossRef] [PubMed]

32. Ahima, R.S.; Flier, J.S. Leptin. Annu. Rev. Physiol. 2000, 62, 4134-4137. [CrossRef]

33. Campfield, L.A.; Smith, F.J.; Burn, P. The OB protein (leptin) pathway-A link between adipose tissue mass and central neural networks. Horm. Metab. Res. 1996, 28, 619-632. [CrossRef] [PubMed]

34. Soukas, A.; Cohen, P.; Socci, N.D.; Friedman, J.M. Leptin-specific patterns of gene expression in white adipose tissue. Genes Dev. 2000, 14, 9639-9680.

35. Shimokawa, I.; Higami, Y. A role for leptin in the antiaging action of dietary restriction: A hypothesis. Aging 1999, 11, 3803-3882. [CrossRef] [PubMed]

36. Hotta, Y.; Nakamura, H.; Konishi, M.; Murata, Y.; Takagi, H.; Matsumura, S.; Inoue, K.; Fushiki, T.; Itoh, N. Fibroblast growth factor 21 regulates lipolysis in white adipose tissue but is not required for ketogenesis and triglyceride clearance in liver. Endocrinology 2009, 150, 46254-46633. [CrossRef]

37. Mikami, K.; Okita, N.; Tokunaga, Y.; Ichikawa, T.; Okazaki, T.; Takemoto, K.; Nagai, W.; Matsushima, S.; Higami, Y. Autophagosomes accumulate in differentiated and hypertrophic adipocytes in a p53-independent manner. Biochem. Biophys. Res. Commun. 2012, 427, 758-763. [CrossRef]

38. Kim, Y.B.; Uotani, S.; Pierroz, D.D.; Flier, J.S.; Kahn, B.B. In vivo administration of leptin activates signal transduction directly in insulin-sensitive tissues: Overlapping but distinct pathways from insulin. Endocrinology 2000, 141, 23282-23339. [CrossRef]

39. Ferré, P.; Foufelle, F. Hepatic steatosis: A role for de novo lipogenesis and the transcription factor SREBP-1c. Diabetes Obes. Metab. 2010, 12, 83-92. [CrossRef]

40. Kammoun, H.L.; Chabanon, H.; Hainault, I.; Luquet, S.; Magnan, C.; Koike, T.; Ferré, P.; Foufelle, F. GRP78 expression inhibits insulin and ER stress-induced SREBP-1c activation and reduces hepatic steatosis in mice. J. Clin. Investig. 2009, 119, 1201-1215. [CrossRef] 
41. Knebel, B.; Haas, J.; Hartwig, S.; Jacob, S.; Köllmer, C.; Nitzgen, U.; Muller-Wieland, D.; Kotzka, J. Liver-specific expression of transcriptionally active srebp-1c is associated with fatty liver and increased visceral fat mass. PLoS ONE 2012, 7, e31812. [CrossRef] [PubMed]

42. Horton, J.D.; Bashmakov, Y.; Shimomura, I.; Shimano, H. Regulation of sterol regulatory element binding proteins in livers of fasted and refed mice. Proc. Natl. Acad. Sci. USA 1998, 95, 59875-59992. [CrossRef] [PubMed]

43. Sekiya, M.; Yahagi, N.; Matsuzaka, T.; Takeuchi, Y.; Nakagawa, Y.; Takahashi, H.; Okazaki, H.; Iizuka, Y.; Ohashi, K.; Gotoda, T.; et al. SREBP-1-independent regulation of lipogenic gene expression in adipocytes. J. Lipid Res. 2007, 48, 1581-1591. [CrossRef]

44. Nisoli, E.; Tonello, C.; Cardile, A.; Cozzi, V.; Bracale, R.; Tedesco, L.; Falcone, S.; Valerio, A.; Cantoni, O.; Clementi, E.; et al. Calorie restriction promotes mitochondrial biogenesis by inducing the expression of eNOS. Science 2005, 310, 314-317. [CrossRef] [PubMed]

45. López-Lluch, G.; Hunt, N.; Jones, B.; Zhu, M.; Jamieson, H.; Hilmer, S.; Cascajo, M.V.; Allard, J.; Ingram, D.K.; Navas, P.; et al. Calorie restriction induces mitochondrial biogenesis and bioenergetic efficiency. Proc. Natl. Acad. Sci. USA 2006, 103, 1768-1773. [CrossRef] [PubMed]

46. Okita, N.; Hayashida, Y.; Kojima, Y.; Fukushima, M.; Yuguchi, K.; Mikami, K.; Yamauchi, A.; Watanabe, K.; Noguchi, M.; Nakamura, M.; et al. Differential responses of white adipose tissue and brown adipose tissue to caloric restriction in rats. Mech. Ageing Dev. 2012, 133, 255-266. [CrossRef] [PubMed]

47. Bruss, M.D.; Khambatta, C.F.; Ruby, M.A.; Aggarwal, I.; Hellerstein, M.K. Calorie restriction increases fatty acid synthesis and whole body fat oxidation rates. Am. J. Physiol. Endocrinol. Metab. 2010, 298, 108-116. [CrossRef]

48. Hao, Q.; Hansen, J.B.; Petersen, R.K.; Hallenborg, P.; Jørgensen, C.; Cinti, S.; Larsen, P.J.; Steffensen, K.R.; Wang, H.; Collins, S.; et al. ADD1/SREBP1c activates the PGC1-alpha promoter in brown adipocytes. Biochim. Biophys. Acta 2010, 1801, 4214-4229.

49. Okita, N.; Tsuchiya, T.; Fukushima, M.; Itakura, K.; Yuguchi, K.; Narita, T.; Hashizume, Y.; Sudo, Y.; China, T.; Shimokawa, I.; et al. Chronological analysis of caloric restriction-induced alteration of fatty acid biosynthesis in white adipose tissue of rats. Exp. Gerontol. 2015, 63, 59-66. [CrossRef]

(C) 2020 by the authors. Licensee MDPI, Basel, Switzerland. This article is an open access article distributed under the terms and conditions of the Creative Commons Attribution (CC BY) license (http://creativecommons.org/licenses/by/4.0/). 